Roberta V. Rada

Eötvös-Loránd-Universität, Budapest

\title{
Die Konzeptualisierung von Grenze im deutschen und ungarischen Mediendiskurs über die Migration im Jahre 2015
}

DOI: 10.14232/fest.bassola.7

\begin{abstract}
Die Migration stellt die europäischen Länder seit 2015 vor immer schwierigere und komplexere Herausforderungen. Sie wird längst nicht mehr nur im Kontext von nationalen Medienkursen sondern auch auf der gesamteuropäischen Ebene, auf der Ebene der Europäischen Union diskutiert. In der ganzen Diskussion und daher auch in den thematisch einschlägigen Mediendiskursen spielen die Landesgrenzen und deren Schutz eine wesentliche Rolle.

Ziel des vorliegenden Beitrags ist es, die Wissensbestände, Denk- und Bewertungsmuster zu ermitteln, die in dem deutschsprachigen und ungarischen Mediendiskurs über die Migration im Zusammenhang mit Grenzen sprachlich vermittelt werden. Dies soll auf der Folie der Diskurslinguistik, im Rahmen einer wortorientierten, deskriptiven linguistischen Diskursanalyse erfolgen. Im Rahmen von quantitativen und qualitativen Untersuchungen sollen die mit dem deutschen Wort Grenze bzw. dem ungarischen Wort határ benannten Konzepte aufgedeckt werden. Die erzielte Analyse versteht sich als international-interlingualer Vergleich von Diskursen.
\end{abstract}

\section{Einleitung: zum Diskurskontext}

Seit 2015 gehört die Migration zu einem der stärksten thematisierten Ereignisse in der Öffentlichkeit. Sie prägt von Anfang an den Alltag der Menschen sämtlicher europäischen Länder, so auch in Ungarn und Deutschland. Dies ist u.a. Ereignissen zu verdanken, wie z.B. der kurzfristige Aufenthalt der Migranten in Ungarn während ihrer Reise aus Südeuropa nach Westeuropa; Szenen, die sich an der ungarischen Grenze, an den ungarischen Bahnhöfen und Landstraßen abgespielt haben; das Ankommen der Flüchtlinge in Deutschland, ihr Emp- 
fang und ihre Aufnahme, das Engagement und der Einsatz von vielen Deutschen bei ihrer Verpflegung und materiellen Unterstützung usw. Für heute ist die Migrationsfrage in erster Linie zu einem politischen Faktor geworden. Die Einstellung der Migrationsfrage gegenüber kann Parteiprogramme und Wahlergebnisse prägen, den Erfolg oder eben Misserfolg von politischen Parteien und/oder Regierungen beeinflussen.

Am 2. Oktober 2016 wurde beispielsweise in Ungarn auf die Initiative der Regierung eine Volksabstimmung zum Thema Migration abgehalten. Die Frage, auf die man antworten musste, lautete: „Akarja-e, hogy az Európai Unió az Országgyülés hozzájárulása nélkül is elöírhassa nem magyar állampolgárok Magyarországra történő kötelező betelepítését?“ (wörtlich: „Möchten Sie, dass die Europäische Union ohne die Zustimmung des ungarischen Parlaments vorschreiben kann, dass nicht ungarische Staatsbürger in Ungarn verbindlich angesiedelt werden können?") Die Volksabstimmung war zwar nicht gültig, weil weniger als die Hälfte der Wahlberechtigten gestimmt haben. Jedoch antworteten $98 \%$ der Wähler mit einem Nein auf die Frage.

Die Migration stellt die europäischen Länder vor immer schwierigere und komplexere soziale, wirtschaftliche und politische Herausforderungen, sie ist längst nicht mehr im Kontext von nationalen Migrationsdiskursen thematisiert sondern zu einem umfassenden Problem auf der gesamteuropäischen Ebene, auf der Ebene der Europäischen Union geworden. Es werden immer wieder neue Lösungsvorschläge unterbreitet bzw. frühere neu diskutiert.

Seit Sommer 2017 erweisen sich im internationalen Maßstab, in den EU-Debatten über die Migration die Grenzen und deren Schutz als zentrale Begriffe. Als Beispiel sollen hier einige Überschriften aus deutschsprachigen und ungarischen Online-Presseprodukten stehen:

(1) Kurz: EU-Grenzschutz soll Migranten an Fahrt übers Mittelmeer hindern (focus.de, 27.05.2018)

(2) Mehr Geld für Grenzschutz statt Quote (tagesschau.de, 12.06.2018)

(3) Titkolja a kormány, miért került 270 milliárdba a határvédelem (hvg.hu, 17.10.2017) („Die Regierung verheimlicht, warum der Grenzschutz 270 Milliarden gekostet hat")

(4) Bejelentéshegyek: Nem is három súlyponti kórház lesz, tolnak pénzt a hulladákszállítóknak, TAO-pénzek és határvédelem (blikk.hu, 19.10. 
2017) ${ }^{1}$ („Berge von Bekanntmachungen: Es wird nicht drei zentrale Krankenhäuser geben, an die Abfalllieferer wird Geld geschoben, TAO-Gelder und Grenzschutz")

Die Migration meint eine Ortsveränderung, die Bewegung von einer größeren Menschenmenge, was mit dem Überschreiten von Landesgrenzen einhergeht. So spielen im Diskurs über die Migration die Grenzen, bzw. generell alles, was an den Grenzen passiert, passieren soll/darf, eine wichtige Rolle.

Vor dem Hintergrund des skizzierten Diskurskontextes wird in diesem Beitrag erzielt, die Wissensbestände, Denk- und Bewertungsmuster zu ermitteln, die in dem deutschsprachigen und ungarischen Mediendiskurs über die Migration im Zusammenhang mit Grenzen sprachlich vermittelt werden. Dies soll auf der Folie der Diskurslinguistik, im Rahmen einer wortorientierten, deskriptiven linguistischen Diskursanalyse (vgl. Spitzmüller 2017) erfolgen. Es sollen die mit dem deutschen Wort Grenze bzw. dem ungarischen Wort határ benannten Konzepte aufgedeckt werden, ohne machtkritisch und damit auch politisch zu sein.

Die erzielte Analyse versteht sich als international-interlingualer Vergleich von Diskursen und soll einen bescheidenen Beitrag zu den vergleichenden Untersuchungen in deutsch-ungarischer Relation leisten. Deutsch-ungarische kontrastive Untersuchungen bilden nämlich einen Forschungsbereich, in dem der Jubilar, Herr Professor Péter Bassola, nicht einfach Hervorragendes geleistet, sondern diesen auch begründet und wesentlich geprägt hat.

\section{Der theoretische Rahmen: Diskurslinguistik, linguistische Diskursanalyse}

In der Diskurslinguistik, die eine neue Disziplin innerhalb der germanistischen Linguistik meint, etablierte sich für heute ein relativ einheitlicher Diskursbegriff. Der Diskurs wird als ein Netzwerk von Texten und Textsequenzen aufgefasst, in dem auf gesellschaftlicher Ebene über einen längeren Zeitraum hin-

Hervorhebungen von mir, R.R. 
weg ein Thema verhandelt wird. Im Sinne von Busse / Teubert (1994) geht es um ein virtuelles Textkorpus, dessen Elemente durch ein gemeinsames Thema zusammengehalten werden. Das Diskursthema ist im Allgemeinen ein gesellschaftlich relevantes Thema, das oft brisant ist. Die Auseinandersetzung mit einem Thema im Diskurs wird ,von mehr oder weniger großen gesellschaftlichen Gruppen getragen" (Gardt 2007: 30). Solche Gruppen, oder auch Einzelpersonen, die das Recht haben im Diskurs mitzureden, am Diskurs teilzuhaben, nennt man Akteure. Nicht jeder Akteur verfügt jedoch über die Möglichkeit, sich im Diskurs Gehör zu verschaffen (vgl. Spitzmüller / Warnke 2011: 61). Wer von einem großen Publikum wahrgenommen werden wird, hat eine bessere Chance, Wissen über das Diskursthema zu vermitteln. Insofern haben Diskurse auch mit Macht zu tun. In den und durch die sprachlichen Handlungen, d.h. Äußerungen und Texte der Akteure wird gesellschaftliches Wissen über das Diskursthema verbreitet. Im Diskurs materialisiert sich, schlägt sich nieder, was „eine Gesellschaft wissen [kann.]“ und auch wie „eine Gesellschaft sagen [kann], was sie zu wissen glaubt" (Roth 2013: 92). Andererseits wird Wissen über ein Diskursthema auch aktiv geprägt. D.h. vorhandenes Wissen bestätigt, in Frage gestellt oder altes Wissen verdrängt, und neues Wissen eingeführt, kurz Wissen ausgehandelt. Das im Diskurs ausgehandelte Wissen soll „handlungsleitend für die zukünftige Gestaltung der gesellschaftlichen Wirklichkeit“ in Bezug auf das Diskursthema wirken (Gardt 2007: 30). Diskurse gelten somit als zentrale Materialisierungs- und Konstitutionsformen von Wissen.

Diskurse sind jedoch für die linguistische Analyse in Form von konkreten Textkorpora greifbar und handhabbar. Allerdings dürfen die beiden Begriffe, d.h. Diskurs und Korpus, nicht identifiziert werden. „Textkorpora dienen dazu, Teilmengen von Diskursen für die linguistische Analyse verfügbar zu machen. Sie sind jedoch weder mit dem Gesamtdiskurs noch mit dem zu untersuchenden Teildiskurs identisch“ deutet Niehr (2014: 33) den Unterschied. Was Linguisten untersuchen können, das sind nur Teildiskurse, kleinere Ausschnitte eines Diskurses, die jeweils durch konkrete Textkorpora repräsentiert sind. Das konkrete Textkorpus wird vom Analysierenden selbst zusammengestellt. Die Korpuszusammenstellung ist zum einen durch eine Reihe von forschungspraktischen Aspekten bedingt, (z.B. Zugänglichkeit, Handhabbarkeit der Texte), zum anderen durch die gezielte Auswahl aus der großen Menge von Texten. Die Auswahl ist wiederum abhängig vom Forschungszweck, hängt mit Vor- 
annahmen, Vorentscheidungen, Intrerpretationen des Analytikers zusammen (vgl. Busse 2013), unterliegt zeitlichen, thematischen o.ä Einschränkungen, und wird durch generelle Kriterien wie Generalisierung, Validität, Reliabilität usw. gesteuert (vgl. Busch 2007).

Der Vergleich von Diskursen kann sich im Sinne von Böke / Jung / Niehr / Wengeler (2000: 12) auf mehrere Staaten (international) oder einen Staat (intranational) beziehen. Vergleicht man thematisch einschlägige Diskurse von mehreren Staaten, so kann der Vergleich mehrere unterschiedliche Sprachen (interlingual) oder lediglich eine Sprache (intralingual) betreffen. Der intranationale Vergleich kann auf einen Diskurs beschränkt sein (intrathematisch) oder sich auf mehrere Diskurse bezogen (interthematisch) sein.

Eine der wichtigsten erkenntnistheoretischen Vorüberlegungen der Diskurslinguistik besagt, dass die Art der sprachlichen Erfassung das Dargestellte prägt und formt. Für abstrakte Gegenstandsbereiche gilt sogar, dass sie in der sprachlich präsentierten Form ohne sprachlichen Zugriff nicht existieren würden. Die Sprache ist in diesem Sinne also gegenstandskonstitutiv (vgl. z.B. Felder 2009, 2013).

Sprachliche Mittel und Muster der Textoberfläche gelten in der Diskurslinguistik als formale Ausprägungen epistemischer Strukturen. Die Analyse von Diskursen erlaubt den Zugriff auf diese Wissensstrukturen, auf gesellschaftliches Wissen. Bei der linguistischen Untersuchung von Diskursen wird aus Beobachtungen in Bezug auf das materiell Sichtbare, also aus Wörtern und Wortverbindungen, Sätzen, syntaktischen Mustern, Textsorten (aber auch Bildern) ausgegangen. Sind solche sprachlichen Strukturen und Phänomene textübergreifend, treten sie statistisch gesehen signifikant häufig im Diskurs auf, gelten sie als musterhaft. Aus diesen rekurrenten sprachlichen Ausdrucksmustern lassen sich dann Rückschlüsse auf Inhaltseitiges, d.h. Wissensbestände, Denkund Bewertungsmuster in Bezug auf relevante Inhalte, Begriffe des Diskurses ziehen (Spitzmüller 2017, Niehr 2014, Bubenhofer 2013).

In der einschlägigen linguistischen Fachliteratur gibt es bis heute keine einheitliche Methode einer diskurslinguistischen Analyse, kein „allgemein angewandtes Verfahren“ (Spitzmüller / Warnke 2011: 121), allerdings liegen „Methodologien" (ebd.: 198), Vorschläge mit unterschiedlichen Analysekriterien, -ebenen und -ansätzen vor. Bei der Erforschung von Diskursen aus linguistischer Perspektive hat sich u.a. die Ebene der lexikalischen Einheiten als zentral 
erwiesen (vgl. Spitzmüller / Warnke 2008, Felder 2012, Spitzmüller 2017). Die wortorientierte Analyse betrifft sowohl Ein- als auch Mehrworteinheiten, wie etwa Kollokationen und Idiome.

Dies bedeutet zugleich, dass lexisch-semantische Untersuchungen und die Interpretation ihrer Ergebnisse in einem neuen theoretischen und methodologischen Rahmen vollzogen werden können.

\section{Das Korpus und das Analyseverfahren}

Diskurslinguistische Untersuchungen basieren, wie oben erwähnt, auf umfangreichen Textkorpora.

Das deutschsprachige Korpus, auf dessen Grundlage die vorliegende Analyse durchgeführt worden ist, versteht sich als Teilkorpus eines größeren, elektronisch gespeicherten, zweisprachigen deutsch-ungarischen Textkorpus, des sog. „Budapester Korpus“ (vgl. dazu Uzonyi 2016). Letzteres ist ein thematisches Korpus, das auf der Basis von Nexis.Lexis mithilfe des Suchausdrucks Migra ${ }^{\star}$ erstellt worden ist, ${ }^{2}$ der in jedem Text des Korpus mindestens einmal vorkommt. Dieses deutschsprachige Migrationskorpus umfasst Texte aus deutschen und österreichischen überregionalen und regionalen Tageszeitungen und Zeitschriften, wie Der Spiegel, Stern, FAZ, SZ, Die Welt, Die Zeit usw. Die Texte repräsentieren die wichtigsten journalistischen Textsorten, es sind hauptsächlich Nachrichten, Berichte, Interviews, Reportagen, es kommen aber auch Leserbriefe vor. Das deutschsprachige Korpus zählt insgesamt 14.629.846 Tokens.

Das ungarische Korpus ist andersartig. Es umfasst ohne thematische Eingrenzung alle Zeitungstexte, die in der Zeitspanne zwischen dem 1. Juli 2014 und dem 31. Dezember 2015 in zwei bedeutenden ungarischen überregionalen Online-Presseprodukten in HVG und MNO veröffentlicht worden sind. HVG ist die eher liberal ausgerichtete Zeitschrift „Heti Világgazdaság“, MNO nennt sich die eher konservative Tageszeitung „Magyar Nemzet“ (zum Korpus vgl.

2 Die Zusammenstellung des Korpus erfolgte im Rahmen der vom DAAD unterstützten Germanistischen Institutspartnerschaft (2012-2016) zwischen dem Germanistischen Seminar der Universität Heidelberg und dem Germanistischen Institut der Eötvös-Loránd-Universität in Budapest von den MitarbeiterInnen und Studierenden beider Universitäten. 
ausführlicher Csatár / Majoros / Tóth 2018: 188-189). Dieses ungarische elektronische Korpus enthält 36.062.001 Tokens.

Von der Größe her können beide Korpora als statistisch repräsentativ (vgl. Stefanowitsch 2005) betrachtet werden.

Die Analyse der beiden Korpora wurde parallel durchgeführt. Im ersten Schritt sind korpus-based quantitative, statistikbasierte Untersuchungen mithilfe des Freeware-Korpusanalyse-Toolkits AntConc durchgeführt worden. Hierbei ist in Anlehnung an Bubenhofer (2009: 100) gefragt worden, „wo wie oft und wie" die Lexeme dt. Grenze bzw. ung. határ in den Korpora auftreten. Durch die Überprüfung der Gebrauchsfrequenz der beiden Wörter, die Zusammenstellung ihres Kookkurrenzprofils, die Auswertung der KWIC-Listen, der Kookkurrenzen und Clusters (teilweise in Anlehnung an Matthias 2018) sind die musterhaft ausgeprägten rekurrenten sprachlichen Ausdrucksmittel auf der Wortebene ermittelt worden. Auf Grund ihrer Interpretation sollen in den beiden Sprachen die mit den Lexemen verbundenen Wissenselemente, also die mit den Wörtern benannten Konzepte eruiert werden.

Im zweiten Schritt erfolgte eine qualitative Analyse von einigen Texten in den beiden Korpora, die sich nach der ersten Lektüre aus inhaltlicher Sicht als relevant erwiesen haben. Durch die Volltextanalysen sollen weitere Aspekte der Konzeptualisierung aufgedeckt werden.

\section{Analyseergebnisse}

\subsection{Lexikostatistische Untersuchungen zum deutschen Lexem Grenze}

Im deutschen Diskurs steht das Lexem Grenze (auch unterschiedliche Flexionsformen des Simplex inbegriffen) im Ranking des Wortvorkommens mit 6468 Treffern an der 251. Stelle.

Von den Wortformtoken im Korpus entfallen 18367 Token auf Wortbildungskonstruktionen, konkreter auf Zusammensetzungen mit der ersten Komponente Grenze-, z.B. Grenzposten, Grenzanlage, Grenzkontrolle und 9272 Token auf solche mit -grenze als Letzt-, meistens Zweitkomponente, z.B. Landesgrenze, Außengrenze, Binnengrenze. Bereits diese Daten zeugen davon, dass Grenze im deutschen Diskurs zu den inhaltlich relevanten Wörtern gehört, mit denen (zumindest) im untersuchten Zeitraum der Diskurs über die Migration geführt wird. 
Auf ihre Häufigkeit hin sind die Wortbildungskonstruktionen stark gestreut. Die mit Abstand häufigsten Zusammensetzungen mit mehr als 1000 Hits sind: Außengrenze $(n)^{3}(1530)^{4}$ und Grenzkontrolle (1226). Diesen folgen Grenzzaun (461), Grenzübergang (440), Grenzübertritt (283), Grenzschutz (256) und Grenzschutzagentur (224) sowie Binnengrenze(n) (125), Grenzschützer (128), und Landesgrenze(n) (102), (synonymisch Landgrenze (53) und Staatsgrenze (89)), die mehr als 100 mal verwendet worden sind. Zu erwähnen sind noch: Grenzbeamten (77), Grenzposten (46), Grenzanlage (29) sowie Grenzüberwachung (19) und Grenzsoldaten (16).

Aufgrund ihrer Referenten, die im jeweiligen Kontext der Lexeme anhand der KWIC-Listen überprüft und bestimmt sind, können die aufgezählten Wortbildungskonstruktionen unterschiedlichen semantischen Domänen zugeordnet werden:

(a) Verwaltung: z.B. Grenzkontrolle, Grenzschutz, Grenzbeamten, Grenzüberwachung

(b) Institutionen: z.B. Grenzschutzagentur

(c) Sicherheitskräfte: z.B. Grenzschützer, Grenzsoldaten

(d) Politik: z.B. Grenzübergang, Grenzübertritt, Binnengrenze(n), Außengrenzen, Land(es)grenze(n), Staatsgrenze

(e) Technische Einrichtungen, Anlagen: z.B. Grenzzaun, Grenzanlage

Die am häufigsten verwendeten Wortzusammensetzungen mit der unmittelbaren Komponente Grenze-/-grenze lassen sich eindeutig den Domänen Verwaltung bzw. Politik zuordnen. Gleichzeitig sind es die beiden Domänen, denen die meisten analysierten zusammengesetzten Wörter zugeordnet werden können.

Die Überprüfung der Referenten hat auch ergeben, dass es unter den untersuchten Wortzusammensetzungen (wie zu erwarten) auch solche gibt, die

(f) auf Verschränkungen und Schranken im übertragenem Sinne Bezug nehmen: z.B. Altersgrenze, Toleranzgrenze, Armutsgrenze, Obergrenze.

3 Bei der Zählung sind die Singular- (Außengrenze) und Pluralformen (Außengrenzen) zusammen betrachtet worden.

4 In Klammern stehen die Trefferzahlen. 
Von diesen fällt, sogar oder geradezu im Vergleich zu den oben analysierten Wörtern, die statistisch gesehen hochfrequente Verwendung von Obergrenze mit 2061 Treffern auf.

Als besonders aufschlussreich erweist sich die Auswertung der häufigsten Kookkurrenzpartner aufgrund der Collocates- (L1, R1) und Clusterlisten (min. 5. max. 10), in denen die ersten 200 Kookkurrenzpartner berücksichtigt worden sind. Diese lassen sich folgenden semantischen Gruppen zuordnen:

- $\quad$ Namen von Ländern bzw. aus solchen abgeleiteten Wörter: mazedonisch, slowenisch, türkisch, österreichisch, Österreich, Deutschland, Ungarn, serbisch, kroatisch, syrisch, griechisch

- Namen von Kontinenten: Europa, Europas, europäisch, inneneuropäisch

- $\quad$ Namen für größere politische Gebilde und Benennungen von wichtigen Verträgen und/oder Beschlüssen von diesen: EU, Schengen

Die oben aufgezählten beziehen sich neben den nationalen Grenzen von Ländern, auch auf die von Kontinenten und politischen Gebilden, nämlich auf solche, die durch die Routen der Flüchtlinge im untersuchten Zeitraum betroffen sind.

Vielfach geht es in den Kookkurrenzpartnern um die Referenz auf die Flüchtlinge und um die Benennung ihrer Handlungen beim Passieren der Grenzen: Menschen, Flüchtlinge, gelangt, überqueren, erreicht, passieren, kommt, stehen, überschritten, stauen, gekommen, gestrandet, pilgern zu Fuß zur Grenze, über die Grenze kommen, die Grenze durchbrechen.

Eine Reihe von Kookkurrenzpartnern benennen Tätigkeiten und Handlungen von anderen, die an den Grenzen auf die dort ankommenden Flüchtlinge ausgerichtet sind: zurückweisen, abweisen, schicken, geschickt, helfen, an der Grenze abgewiesen werden.

Unter den Kookkurrenzpartnern gibt es auch Lexeme, die darauf Bezug nehmen, was an den Grenzen administrativ vollzogen wird: schützen, kontrollieren, kontrolliert, sichern, offene, öffnen, geöffnet, öfnet, schließt, dichtmachen, dichtgemacht, geschlossen, dicht, Kontrolle an der Grenze.

Sehr häufig kommen in der syntaktischen Umgebung des Lexems Grenze auch Possessivpronomina vor: seiner, seine, ihrer, unserer. 
Die bereits präsentierten Daten und ihre Auswertung legen eine intensionale Bedeutungsvielfalt des Lexems Grenze im deutschsprachigen Mediendiskurs über die Migration nahe.

Im Diskurs werden abhängig von verschiedenen Ko- und Kontexten folgende Begriffsinhalte des Lexems Grenze aktiviert:

(a) politische Dimension: die Grenze als Linie oder Geländerstreifen, die/der politische Gebilde, jedoch nicht nur Länder, Staaten sondern auch von Verbunden von Ländern (wie Europäische Union) von einander trennt

(b) Verwaltungsdimension, Dimension der Administration: der Grenzbetrieb

(c) technische Dimension: die Anlagen, die an den Grenzen teils zum Zwecke der Markierung teils zum Zwecke der Durchführung der administrativen Aufgaben an den Grenzen errichtet werden sowie

(d) Beschränkung, Begrenzung: in erster Linie bezogen auf die Zahl der Flüchtlinge und auf die beschränkte Aufnahmefähigkeit des Landes, z.B. Deutschland steht an der Grenze ihrer Aufnahmefähigkeit, stößt an seine Grenzen, keine Obergrenze für Flüchtlinge.

\subsection{Lexikostatistische Untersuchungen zum ungarischen Lexem határ}

Eine vergleichbare Recherche im ungarischen Korpus war mit einer Reihe von Schwierigkeiten verbunden. Zum einen rühren sie daher, dass das ungarische Korpus kein thematisches Korpus ist (vgl. oben), zum anderen hängen sie mit den morphologischen Besonderheiten der ungarischen Sprache zusammen.

Versucht man mit der Analysesoftware AntConc (analog zum Deutschen) eine KWIC-Liste zum ungarischen Suchausdruck határ zu generieren, so kann ausschließlich das Vorkommen des Simplex határ ohne morphologische Flexionsformen ermittelt werden, zumal im Ungarischen die Kategorie Kasus am Wortstamm mithilfe von Affixen markiert wird, z.B. határt (Akkusativ), határnak (Dativ). Gibt man határ ${ }^{\star}$ als Suchausdruck ein, erscheinen nicht nur die flektierten Formen des Lexems sondern auch substantivische Wortbildungskonstruktionen, wie határátkelő („Grenzübertretende(r)”), határőrség („Grenz-

\footnotetext{
5 In Klammern stehen die wörtlichen Übersetzungen, um die morphologische Struktur der ungarischen Wörter sichtbar zu machen. An manchen Stellen wird gelegentlich die Bedeutung der
} 
wache”), határido” („Grenzzeit“ i.S.v. 'Termin'), határkerités („Grenzzaun”) usw. oder Ableitungen der unterschiedlichsten Wortart, wie határozat ('Entscheidung'), határozottság ('Entschiedenheit'), határoz ('entscheiden, beschließen'), határolódik ('sich abgrenzen'), határozott ('entschlossen' als Adjektiv), határozottan ('entscheidend' als Adverb). Insbesondere die Ableitungen produzieren viele irrelevante Daten. Aus diesen Gründen bzw. wegen einer besseren Vergleichbarkeit mit dem deutschen Korpus wurden alle Wortzusammensetzungen mit határ- als erster Komponente sowei die beiden häufigsten mit -határ als Letztkomponente, nämlich országhatár („Landesgrenze”) und államhatár („Staatsgrenze”), berücksichtigt. Zugleich wurde auch überprüft, ob es sich um Wortverwendungen im thematischen Kontext der Migration handelt. Selbst wenn diese statistischen Angaben mit denen des deutschen Migrationskorpus nur bedingt vergleichbar sind, sind sie imstande, ein zuverlässiges Bild über die Konzeptualisierung von dem Lexem határ im ungarischen Mediendiskurs zu vermitteln.

Im ungarischen Korpus erweisen sich folgende Wortzusammensetzungen als am frequentesten: határkerités („Grenzzaun”) (3732), határzár (wörtlich „Grenzschloss“, meint aber einen Grenzzaun) (1755), határátkelo/-lés/-hely („Grenzüberquerende(r)/Grenzüberqueren/-überquersort”) ${ }^{6}$ (1352), határátlépés/-pő („Grenzübertretung/Grenzübertretende(r)”) (1001), határsértő/ -sértés („Grenzverleztzende(r)/-verletzung”) (996), határőr/-ség („Grenzwächter/-wache”) (965), határellenőrzés („Grenzkontrolle”) (613), határszakasz („Grenzstrecke”) (575), határvédelem/-védelmi („Grenzschutz“ bzw. das davon abgeleitete Adjektiv) (419), határrendész/-et („Grenzwächter/-wache”) (306), országhatár („Landesgrenze”) (240), államhatár („Staatsgrenze”) (209), határállomás („Grenzstation”) (93), határvonal („Grenzlinie”) (78).

Sie können, ähnlich wie die deutschen Beispiele, den folgenden semantischen Domänen zugeordnet werden:

(a) Verwaltung: z.B. határellenőrzés, határvédelem, határátlépő, határátkelö/-lés

Wörter angegeben, weil in bestimmten Fällen die Bedeutungsangabe und nicht die Ermittlung der morphologischen Struktur den Nachvollzug des Gedankenganges ermöglicht.

6 Die morphologisch verwandten Formen konnten aus technischen Gründen nur zusammen gezählt werden. 
(b) Sicherheitskräfte: z.B.: határőr/-ség, határrendész/-et

(c) Politik: z.B. határvonal, határszakasz, országhatár, államhatár

(d) Technische Einrichtungen, Anlagen: határállomás, határzár, határkerités, határátkelöhely.

Im Ungarischen sind aufgrund der Analyse der Wortzusammensetzungen mit der Erstkomponente határ- die Domänen Technische Einrichtung, Sicherheitskräfte und Verwaltung am stärksten belegt. Im Unterschied zum deutschen ist im ungarischen Mediendiskurs auch noch eine Domäne präsent, nämlich

(e) Rechtswesen: határsértő/-tés („Grenzverletzung/-verletztende(r)”).

Unter den untersuchten Wortzusammensetzungen gab es keine, die, wie im deutschen Diskurs, auf Verschränkungen und Schranken im übertragenen Sinne referiert.

Die anhand der Collocates- und Cluster-Listen ermittelten allerhäufigsten Kookkurrenzpartner des substantivischen Lexems határ (die ersten 200 im Ranking)

a) beziehen sich auch im Ungarischen auf die Namen von Ländern und politischen Gebilden, die (nicht nur südlichen) Grenzländer Ungarns oder die Herkunft der Flüchtlinge oder aber die Flüchtlingsrouten betreffen: magyar („ungarisch”), szerb („serbisch”), szíriai („syrisch”), osztrák („österreichisch”), horvát („kroatisch”), török („türkisch”), ukrán („ukrainisch”), macedón („mazedonisch”), szlovén („slowenisch”), görög („griechisch”), német („deutsch”), orosz („russisch”), pakisztáni („pakistanisch”), líbiai („lybisch”), szerb („serbisch”), európai uniós („der Europäischen Union”), külső „äußere”), schengeni („Schengener”), magyar-szerb („ungarisch-serbisch”), osztrák-szlovén („österreichisch-slowenisch”), szerb-horvát („serbisch-kroatisch”), (határ) szerbiai oldala („serbische Seite (der Grenze)")

b) benennen in Form von synonymischen Ausdrücken explizit den Schutz der Grenzen: védelem („Schutz“ und ihre morphologischen Formen védelmét/-ben/-hez/-ről/-nek), megvédés („Verteidigung”), őrzésében („,in/ an der Kontrolle”), biztositás („Sicherung”), megerősítése („Stärkung”), 
őrizete („Schutz”), lezárása („Abriegelung”). Mit diesen Wörtern im Zusammenhang, um diese zu attribuieren, treten oft Adjektive auf hatékony („wirksam”), hermetikus („hermetisch”), fizikai („physisch/physikalisch")

c) sind richtungsbezogene, deiktische Ausdrücke bzw. Adverbien und Postpositionen: közelében („in der Nähe”), innenső („diesseitige”), túloldali (jenseitige”), irányába („in Richtung (Grenze)”), mentén („,entlang”)

d) benennen Himmelsrichtungen: déli („,südlich”), keleti („östlich”), nyugati („westlich”)

e) bezeichnen Handlungen, die an den Grenzen vollzogen werden: átlépése („Übertritt”), megnyitása („Öffnung”), lezárása („Abriegelung”), határ hermetikus lezárása („hermitische Abriegelung der Grenze”), határ mentén épitett kerités („der entlang der Grenze gebaute Zaun”), gyorstelepitésü drótakadály kiépitése („Ausbau des schnell angelegten Stachelhindernisses")

Aufgrund der Auswertung dieser lexikostatistischen Angaben lassen sich im ungarischen Mediendiskurs teils ähnliche intensionale Schattierungen des Lexems határ wie im deutschen Diskurs erarbeiten:

(a) politische Dimension: die Grenze als Linie oder Geländerstreifen, die/ der politische Gebilde, jedoch nicht nur Länder, Staaten sondern auch Länderverbunde (wie Europäische Union) von einander trennt

(b) Verwaltungsdimension, Dimension der Administration: der Grenzbetrieb mit besonderer Perspektivierung des Schutzes der ungarischen Grenzen, die zugleich die Außengrenzen der EU bedeuten (vgl. insbesondere die Kookkurrenzpartner)

(c) technische Dimension: die Anlagen, die an den Grenzen in erster Linie zum physischen Schutz der Grenzen und nicht zum Zweck der Durchführung der administrativen Aufgaben an den Grenzen errichtet werden (vgl. insbesondere die Kookkurrenzpartner)

(d) Beschränkung, Begrenzung im übetragenen Sinne, vertreten durch die Kollokation felső határ („Obergrenze”). 
Bei der Konzeptualisierung von határ spielt auch die

(e) geografische Dimension eine Rolle: vertreten durch die Namen von Himmelsrichtungen und die richtungsbezogenen Wörter. Dies hängt damit zusammen, dass im Ungarischen die Bewegung der Migranten in der Umgebung der Grenze besonders stark perspektiviert wird.

Im ungarischen Korpus verfügt das Lexem határzár ('Grenzzaun') mit Abstand über die größte Vorkommenshäufigkeit (vgl. oben). Grenzzaun wird auch im deutschen Migrationsdiskurs sehr frequent gebraucht. Deshalb wurden im nächsten Schritt die Konzepte, die mit den Lexemen határzár bzw. Grenzzaun versprachlicht worden sind, im Rahmen einer qualitativen Volltextanalyse untersucht, um dadurch spezifischere Aspekte der Konzeptualisierung von határ und Grenze erfassen zu können. Auch in den Volltextanalysen wurde sich auf die sprachlichen Ausdrucksmuster auf der Wortebene konzentriert.

\subsection{Volltextanalysen: dt. Grenzzaun}

Im deutschen Mediendiskurs über die Migration können hinsichtlich der Konzeptualisierung von Grenzzaun drei Ausdrucksmuster hervorgehoben werden.

(a) Zum einen sind es wertende und affektive Wörter und Ausdrücke bzw. solche, die Wertungen und/oder Gefühle benennen, z.B. schockiert, überrascht, falsch, empört, wütende, fremdenfeindlich, äußerte sich kritisch, sorgt für Aufregung.

Zum einen wird mit Hilfe dieser Ausdrücke nicht der Grenzzaun als Objekt, sondern die ungarische Regierung oder der ungarische Ministerpräsident Viktor Orbán negativ bewertet:

(5) Soll Deutschland etwa Grenzzäune bauen lassen wie Ungarns fremdenfeindlicher Premierminister Viktor Orbán? (Der Spiegel, 02.10. 2015)

Zum anderen spiegeln sie die Reaktionen der Nachbarländer Ungarns, insbesondere von Serbien und Österreich, wider, zumal sie am ehesten und unmittelbarsten davon betroffen wird, was an den ungarischen Grenzen mit den Migranten passiert. 
(6) Serbien reagiert empört. Andere serbische Medien empörten sich über die Ankündigung, eine chinesische Mauer zwischen den Staaten zu bauen. (Der Standard, 19.06. 2015)

(7) Serbiens Ministerpräsident, Aleksandar Vugchigca, zeigte sich über den ungarischen Plan „schockiert und überrascht“. (Die Presse, 19.06. 2015)

(8) Auch Österreichs Bundespräsident, Heinz Fischer, äußerte sich kritisch zu dieser Maßnahme, mit der Ungarns Regierung die illegale Einreise von Migranten verhindern möchte. (...) Das sei ein „Schritt in die falsche Richtung“, sagte Fischer. (Die Presse, 19.06.2015)

(9) „Die Idee, das Flüchtlingsproblem mit Zäunen innerhalb Europas zu lösen, halte ich für eine, der ich gar nichts abgewinnen kann“, betonte der Bundespräsident. (Die Presse, 19.06.2015)

(b) Zweitens geht es um Ausdrücke, die auf (oft konkrete, namentlich genannte, in der Geschichte berühmt/berüchtigt gewordene) technische Objekte bzw. Bauten referieren, die hinsichtlich ihrer Funktion mit dem ungarischen Grenzzaun vergleichbar sind: Mauer, Berliner Mauer, Vorhänge, Eiserner Vorhang, KZ Auschwitz. Parallelen und Gegenüberstellungen dienen dazu, durch das Aktivieren von negativen Assoziationen zu den erwähnten Objekten die Handlungen und die Einstellung der ungarischen Regierung den Migranten gegenüber zu verurteilen:

(10) Es sei jedem Staat überlassen, wie er seine Grenzen schütze, sagte am Donnerstag eine Sprecherin der EU-Kommission. „Die EU unterstützt jedoch nicht den Bau von Zäunen“, erklärte sie. „Wir haben gerade erst die Mauern in Europa niedergerissen, wir sollten sie nicht wieder aufbauen." (Die Presse 19.06.2015)

(11) 1989 war Ungarn das erste kommunistische Land, das den Eisernen Vorhang abriss, die mit Wachtürmen bewehrte Sperranlage an der Grenze zu Österreich. Jetzt, 26 Jahre später, soll nach dem Willen des rechtspopulistischen ungarischen Ministerpräsidenten Viktor Orbán ein neuer Eiserner Vorhang entstehen, diesmal an der Südgrenze zu Serbien. Der vier Meter hohe, 175 Kilometer lange Metallzaun soll nicht Inländer vom Verlassen, sondern Ausländer vom Betreten des Landes abhalten. (Der Spiegel, 9.26.2015) 
(12) Belgrad werde seinerseits sicher nicht Mauern an seinen Staatsgrenzen errichten und eingeschlossen wie in Auschwitz leben, erklärte Serbiens Ministerpräsident Aleksandar Vučić. Serbien sei nur ein Transitland und habe keine Schuld an dem Flüchtlingsstrom, der über die EU-Länder Griechenland und Bulgarien nach Serbien ziehe. (Der Standard 19.06.2015)

(c) Die Thematik des Grenzschutzes wird in einem weiteren europäischen Kontext betrachtet und nach Lösungen der Migrationsproblematik auf der EU-Ebene gesucht. Davon zeugen die Ausdrücke wie europäische Grundrechtcharta, Asylsystem überprüfen, die Verteilung der Flüchtlinge, Quoten, Europa der Grenzen, Europa plant nun, EU-Länder, Drittstaaten, Staats- und Regierungschefs der EU, die Außengrenze künftig besser kontrollieren.

(13) Doch die Zuversicht, dass Europa einer Lösung der Flüchtlingskrise auch nur nähergerückt ist, will sich außerhalb Brüssels nicht recht einstellen. Flüchtlingshelferin Stroux sagt: „Ich sehe momentan nichts als Stacheldrahtzäune, Schiffswracks und Flüchtlinge, die hungern." Auch andere EU-Staaten finden keine Antworten auf die Krise. Sie setzen auf Wasserwerfer, Pfefferspray und Grenzkontrollen. Die Flüchtlingsfrage spaltet Europa noch mehr als die Eurokrise. (Der Spiegel, 26.09.2015)

\subsection{Volltextanalysen: ung. határzár}

Im ungarischen Mediendiskurs dominieren größtenteils andersartige sprachliche Ausdrucksmuster auf der Wortebene.

(a) Zunächst fällt auf, dass sich mehrere referenzidentische Ausdrücke für den Grenzzaun eingebürgert haben. Neben dem frequentesten határzár (wörtlich „Grenzschloss”) verwendet man auch határkerités („Grenzzaun”), kerités („Zaun”), müszaki zár („technisches Schloss”), gyorstelepitésü drótakadály („schnell angelegtes Stachelhindernis”), von denen einige als verschönernd-verschleiert gelten.

(b) Der ungarische Mediendiskurs ist durch das häufige Vorkommen von Hochwertwörtern geprägt z.B. kötelezettség („Verpflichtung”), biztonság („Si- 
cherheit”), szabadság („Freiheit”), életforma („Lebensform”). Sie drücken nicht nur nationale, sondern auch europäische Werte aus, die wegen der Migranten in Gefahr sind: veszélybe került („geriet in Gefahr”), veszélyben van („ist in Gefahr”), a szabadság forgott kockán („Die Freiheit stand auf dem Spiel”), elvegyék a szabadságunkat („unsere Freiheit wegnehmen”), und die daher verteidigt werden müssen: szabadságunk megvédése („Verteidigung unserer Freiheit”), az életformánkat védjük („wir verteidigen unsere Lebensform”).

(14) A kormányfő szerint a jelenlegi bevándorlási hullámmal veszélybe került az európai életforma, amelyet meg kell védeni, márpedig Európát csak akkor lehet megtartani olyannak, amilyen, ha nem engedünk be mindenkit. (HVG, 15-09.2015)

(„Laut dem Regierungschef ist durch die gegenwärtige Migrationswelle die europäische Lebensform in Gefahr geraten, sie muss verteidigt werden. Europa kann jedoch in seiner jetzigen Form erst beibehalten werden, wenn wir niemanden hereinlassen." $)^{7}$

(c) Durch eine Reihe von sprachlichen Ausdrucksmitteln werden die Funktionen des Grenzzauns verbalisiert.

- Schutz/Verteidigung der Grenzen und Außengrenzen der EU: határaink védelme („Schutz unserer Grenzen”), a schengeni határok védelme („Schutz der Schengener Grenzen”), megvédeni az uniós határokat („die Grenzen der Union schützen")

(15) Pintér Sándor kijelentette: a határzárt az államhatár védelme indokolta, ez nemzetbiztonsági érdek. (HVG, 30.09.2015)

(„Sándor Pintér erklärte: Der Grenzzaun ist durch den Schutz der Staatsgrenze begründet, es ist im Interesse der nationalen Sicherheit.“)

Übersetzungen von mir, R. R. 
aber auch

- Schutz gegen die Migranten, die eine Bedrohung, eine Gefahr bedeuten. Teils schlägt sich das sprachlich in Metaphern nieder: z.B. megfékezze az illegális bevándolók áramát („den Migrantenstrom bremsen”), áradat („Flut”), migránsáradat („Migrantenflut”) (FLUT-Metapher), ostrom („Sturm”) (GEBÄUDE-Metapher $)^{8}$. Teils dadurch, dass die Migranten durch illegales, gesetzwidriges Verhalten konzeptualisiert werden: megpróbálnak átszökni („sie versuchen über die Grenze zu schleichen”), határzár illegális átlépése („der illegale Übertritt des Grenze”), büncselekmény („Verbrechen”), amiért börtön jár, („wofür man eine Gefängnisstrafe erhält”).

(16) A segítséget joggal várják el, de ez nem azonos azzal, hogy „idejövök, és megpróbálok elvenni az európaiaktól valamit, amiért azok megdolgoztak." (HVG, 15.09.2015)

(„Die Hilfe wird mit Recht erwartet, aber das bedeutet nicht ,ich komme hierher und versuche den Europäern wegzunehmen, wofür sie gearbeitet haben.")

Die Migranten gefährden also nicht nur Ungarn, sondern auch Europa, deren Einwohner und Werte, die verteidigt werden müssen. Dies kann durch die Grenzen des Landes bzw. deren Verteidigung gewährleistet werden. Somit werden die Grenzen und der Grenzzaun als die wichtigsten Verteidigungslinien bzw. -mittel konzeptualisiert.

d) Auch im ungarischen Mediendiskurs spielen Ausdrücke eine Rolle, die technische oder Bauobjekte benennen, die in anderen Ländern, z.B. in Estland und Lettland, in Marokko, in Griechenland und Bulgarien zum Schutz der Landesgrenzen und/oder der EU-Außengrenzen und/oder Kontinentgrenzen (Afrika) im Kontext der Migration errichtet worden sind: falak („Mauern”), kerités („Zaun”), szögesdrót kerítés („Stacheldrahtzaun”), vasfüggöny („Eiserner Vorhang"). Sie werden als Beispiel herangezogen, um die Errichtung des ungarischen Grenzzauns als begründete Handlung zu legitimieren:

Zu den Metaphern des ungarischen Mediendiskurses über die Migration vgl. Csatár / Majoros / Tóth 2018. 
(17) Nem csak a magyarok építenek kerítést (MNO, 3.09.2015)

(„Es sind nicht nur die Ungarn, die einen Zaun bauen.")

(18) Európában több ország is falakat emel, hogy megfékezze az illegális bevándorlók áradatát. (MNO, 3.09.2015)

(„Mehrere Länder in Europa errichten Mauern, um die Flut der illegalen Einwanderer zu bremsen.")

\section{Zusammenfassung}

Die Konzeptualisierung der Grenze weist in den beiden Mediendiskursen neben zahlreichen Gemeinsamkeiten auch relevante Unterschiede auf. Dies kann auf die unterschiedlichen Einstellungen gegenüber der Migrationsfrage, auf die unterschiedliche Konzeptualisierung von Migranten zurückgeführt werden.

Im ungarischen Mediendiskurs sieht man in der Migration eine Gefahr, vor der das Land und die Einwohner des Landes geschützt/verteidigt werden müssen. Ein effektives Mittel dieses Schutzes stellen die Grenzen dar (politische Dimension und Verwaltungsdimension). Die Grenzen werden eher als etwas Konkretes, materiell Wahrnehmbares aufgefasst: Etwas, was man überqueren, überschreiten kann, was man durch Bauten und technische Errichtungen physisch abgeriegeln kann, wo Grenzsoldaten tätig sind usw. (geografische und technische Dimension). Der Schutz der Landesgrenzen soll die Sicherheit garantieren. Der Grenzschutz wiederum wird als die innere Angelegenheit des Landes, als Recht und Pflicht der ungarischen Regierung (Rechtsdimension), als Verpflichtung den eigenen Landsleuten, Ungarn, aber auch den Europäern und Europa gegenüber aufgefasst, bei dem in erster Linie die nationalen Interessen berücksichtigt werden.

Im deutschsprachigen Mediendiskurs scheinen die Grenzen eher symbolisch zu sein. Zum einen, weil die Landesgrenzen von Deutschland als Binnengrenzen der EU gelten, also nicht - im Unterschied zu Ungarn - wie Außengrenzen geschützt werden müssen (politische Dimension). Zum anderen wird in der Migration keine Gefahr, Bedrohung gesehen, vor der man Schutz braucht. Die Kontrolle der Migranten an den deutschen und österreichischen Grenzen gilt als Formalität (Verwaltungsdimension). Die Lösung der Migrationsproblematik erwartet man auf der Ebene der EU und sie wird auf keinen Fall in der Abriegelung der Landesgrenzen durch Mauern, Zäune, usw. gesehen. 


\section{Literatur}

Böke, Karin / Jung, Matthias / Niehr, Thomas / Wengeler, Martin (2000): Vergleichende Diskurslinguistik. Überlegungen zur Analyse national heterogener Textkorpora. In: Niehr, Thomas / Böke, Karin (Hrsg.): Einwanderungsdiskurse. Vergleichende diskurslinguistische Studien. Wiesbaden: Westdeutscher Verlag, 11-36.

Bubenhofer, Noah (2009): Sprachgebrauchsmuster. Korpuslinguistik als Methode der Diskurs- und Kulturanalyse. Berlin / New York: de Gruyter.

Bubenhofer, Noah (2013): Quantitativ informierte qualitative Diskursanalyse. Korpuslinguistische Zugänge zu Einzeltexten und Serien. In: Roth, Kersten Sven / Spiegel, Carmen (Hrsg.): Angewandte Diskurslinguistik. Felder, Probleme, Perspektiven. Berlin: Akademieverlag, 109-134.

Busch, Albert (2007): Der Diskurs: ein linguistischer Proteus und seine Erfassung. Methodologische und empirische Gütekriterien für die sprachwissenschaftliche Erfassung von Diskursen und ihrer lexikalischen Inventare. In: Warnke, Ingo (Hrsg.): Diskurslinguistik nach Foucault. Theorie und Gegenstände. Berlin / New York: de Gruyter, 141-167.

Busse, Dietrich (2008): Diskurslinguistik als Epistemologie - Das verstehensrelevante Wissen als Gegenstand linguistischer Forschung. In: Warnke, Ingo H. / Spitzmüller, Jürgen (Hrsg.): Methoden der Diskurslinguistik. Sprachwissenschaftliche Zugänge zur transtextuellen Ebene. Berlin: de Gruyter, 57-87.

Busse, Dietrich (2013): Linguistische Diskurssemantik. In: Busse, Dietrich / Teubert, Wolfgang (Hrsg.): Linguistische Diskursanalyse: neue Perspektiven. Wiesbaden: Springer, 31-54.

Busse, Dietrich / Teubert, Wolfgang (1994): Ist Diskurs ein sprachwissenschaftliches Objekt? Zur Methodenfrage der historischen Semantik. In: Busse, Dietrich / Hermanns, Fritz / Teubert, Wolfgang (Hrsg.): Begriffsgeschichte und Diskursgeschichte. Methodenfragen und Forschungsergebnisse der historischen Semantik. Opladen: Westdeutscher Verlag, 10-28.

Csatár, Péter / Majoros, Krisztián / Tóth, Máté (2018): Die metaphorische Repräsentation der Migrationskrise von 2014-2015 in zwei führenden ungarischen Online-Zeitschriften. Eine Bestandaufnahme. In: Kovács, Kálmán / Vortisch, Verena (Hrsg.): Jahrbuch der ungarischen Germanistik 2017. Budapest / Bonn: GuG / DAAD, 187-210. 
Felder, Ekkehard (2009): Sprache - das Tor zur Welt!? Perspektiven und Tendenzen in sprachlichen Äußerungen. In: Felder, Ekkehard (Hrsg.): Sprache. Im Auftrag der Universitätsgesellschaft Heidelberg. Berlin u.a.: Springer Verlag (Heidelberger Jahrbücher 53), 13-57.

Felder, Ekkehard (2012): Pragma-semiotische Textarbeit und der hermeneutische Nutzen von Korpusanalysen für die linguistische Mediendiskursanalyse. In: Felder, Ekkehard / Müller, Marcus / Vogel, Friedemann (Hrsg.): Korpuspragmatik. Thematische Korpora als Basis diskurslinguistischer Analysen. Berlin / New York: de Gruyter, 115-174.

Felder, Ekkehard (2013): Linguistische Diskursanalyse im Forschungsnetzwerk „Sprache und Wissen“. In: Keller, Reiner / Schnider, Werner / Viehöver, Willy (Hrsg.): Diskurs - Sprache - Wissen. Interdisziplinäre Beiträge zum Verhältnis von Sprache und Wissen in der Diskursforschung. Wiesbaden: VS Verlag, 167-198.

Gardt, Andreas (2007): Diskursanalyse. Aktueller theoretischer Ort und methodische Möglichkeiten. In: Warnke, Ingo (Hrsg.): Diskurslinguistik nach Foucault. Theorie und Gegenstände. Berlin / New York: de Gruyter, 27-53. Mathias, Alexa (2018): Lexik und Legitimation in rechtspopulistischen Bewegungen: Korpuslinguistische Befunde aus Facebook-Postings von Pegida-Sympathisanten. In: Muttersprache 1/2018, 41-51.

Niehr, Thomas (2014): Einführung in die linguistische Diskursanalyse. Darmstadt: WBG.

Roth, Kersten Sven (2013): Qualitativ-diskurspragmatische Vorwissensanalyse. Angewandte Diskurslinguistik im institutionellen Wissenstransfer aus Politik, Wirtschaft und Wissenschaft. In: Roth, Kersten Sven / Spiegel, Carmen (Hrsg.): Angewandte Diskurslinguistik. Felder, Probleme, Perspektiven. Berlin: Akademieverlag, 89-108.

Spitzmüller, Jürgen (2017): Deskriptive, linguistische Diskursanalyse. In: Der Deutschunterricht 6/2017, 44-53.

Spitzmüller, Jürgen / Warnke, Ingo H. (2011): Diskurslinguistik. Eine Einführung in Theorien und Methoden der transtextuellen Sprachanalyse. Berlin / Boston: de Gruyter.

Stefanowitsch, Anatol (2005): Quantitative Korpuslinguistik und sprachliche Wirklichkeit. In: Solte-Gresser, Christiane et. al. (Hrsg.): Von der Wirklich- 
keit zur Wissenschaft: Aktuelle Forschungsmethoden in den Sprach-, Literatur- und Kulturwissenschaften. Hamburg: LIT-Verlag, 141-155.

Uzonyi, Pál (2016): Deutsche und ungarische Geschichte. Zur Erforschbarkeit ihrer Berührungspunkte mit Hilfe von elektronischen Korpora. In: Beßlich, Barbara / Felder, Ekkehard (Hrsg.): Geschichte(n) fiktional und faktual. Literarische und Diskursive Erinnerungen im 20. und 21. Jahrhundert. Berlin u. a.: Peter Lang, 153-164.

Warnke, Ingo H. / Spitzmüller, Jürgen (2008): Methoden und Methodologie der Diskurslinguistik - Grundlagen und Verfahren einer Sprachwissenschaft jenseits textueller Grenzen. In: Warnke, Ingo H. / Spitzmüller, Jürgen (Hrsg.): Methoden der Diskurslinguistik. Sprachwissenschaftliche Zugänge zur transtextuellen Ebene. Berlin: de Gruyter (Linguistik - Impulse \& Tendenzen), 3-54. 